\title{
The Costs of Warfarin Underuse and Nonadherence in Patients with Atrial Fibrillation: A Commercial Insurer Perspective
}

\author{
Julian P. Casciano, BS; Zenobia J. Dotiwala, MS; Bradley C. Martin, PharmD, PhD; \\ and Winghan Jacqueline Kwong, PharmD, PhD
}

\begin{abstract}
BACKGROUND: Atrial fibrillation (AF) imposes a substantial clinical and economic burden on the U.S. health care system. Despite national guidelines that recommend oral anticoagulation for stroke prevention, the literature consistently reports its underuse in AF patients with moderate to high stroke risk.
\end{abstract}

OBJECTIVE: To assess the economic burden of underuse and nonadherence of warfarin therapy among patients with nonvalvular AF in a commercially insured population.

METHODS: Claims data between January 2003 and December 2007 from the Thomson Reuters MarketScan Research Database were used. Patients diagnosed with nonvalvular AF who were continuously enrolled for at least 12 months prior to and 2 months following their diagnosis, who had a $\mathrm{CHADS}_{2}$ score $\geq 2$, and were not at high risk of bleeding (ATRIA score $<5$, HEMORR ${ }_{2}$ HAGE score $<4$, and HAS-BLED score $<3$ ) at baseline were included. Patients were followed for up to 18 months after the AF diagnosis date to assess the level of warfarin utilization. Health care resource utilization and cost during follow-up among patients with the proportion of days covered (PDC) by warfarin $>0.8$ (high) and $\leq 0.8$ (low) versus patients with no warfarin exposure were assessed. Multivariate negative binomial regressions and generalized linear models were used to estimate differences in resource utilization and cost, respectively.

RESULTS: Of the 13,289 subjects included in this analysis, $47 \%$ had no warfarin exposure; $31.5 \%$ had low PDC; and $21.5 \%$ had high PDC. The rates of ischemic stroke and transient ischemic attack (per 100 patient-years) were significantly lower for the groups that had high and low PDCs as compared with the group with no warfarin exposure $(P<0.001)$. Multivariate analysis showed that patients with high PDC were $27 \%$ less likely $(P<0.001)$ to incur hospitalizations, and $16 \%$ were less likely $(P=0.019)$ to incur emergency room visits than patients who did not receive warfarin, but the differences between low PDC patients and no warfarin exposure were not significant. Although both low and high PDC were associated with lower all-cause inpatient cost $(P<0.001)$, only high PDC was associated with a lower post-index all-cause total cost $(P<0.001)$ compared with no warfarin exposure.

CONCLUSION: Our results confirm that underutilization and nonadherence of warfarin among nonvalvular AF patients is both prevalent and costly. Warfarin use among patients with moderate to high stroke risk and low to moderate bleed risk demonstrated a stroke benefit without a significant increase in intracranial hemorrhage. Adherence to oral anticoagulant therapy was associated with a significant reduction in inpatient service use and total health care cost. Improving adherence to oral anticoagulation is important to attaining the clinical and economic benefits of therapy.

J Manag Care Pharm. 2013;19(4):302-16

Copyright $\odot 2013$, Academy of Managed Care Pharmacy. All rights reserved.

\section{What is already known about this subject}

- Atrial fibrillation (AF) is estimated to affect more than 3 million U.S. adults. It is associated with significant increase in the likelihood of stroke, resulting in a substantial clinical and economic burden.

- The efficacy of warfarin for stroke prevention in AF patients is well established, but the literature consistently shows that it is underused even in patients at high risk of stroke.

- Maintaining adherence to warfarin therapy is challenging. Previous data have shown that 1 in 4 AF patients discontinue warfarin therapy within the first year of receiving their initial prescriptions

\section{What this study adds}

- High warfarin adherence (proportion of days covered $>80 \%$ ) was associated with fewer inpatient admissions and emergency room visits and lower total health care cost, compared with no use of warfarin at all. These data underscore the economic and clinical benefits of improving oral anticoagulant use in AF patients.

A trial fibrillation (AF) is the most common type of cardiac arrhythmia, and it affects more than 3 million U.S. adults. ${ }^{1}$ AF is responsible for $15 \%$ to $20 \%$ of all strokes ${ }^{2}$ and confers almost 5 times the risk of stroke and 1.9 times the risk of death versus patients without $\mathrm{AF}^{2,3}$ Total direct cost related to AF treatment in the United States is reported to be more than $\$ 6$ billion annually. ${ }^{4}$ The overall economic burden of stroke from a societal perspective, including both direct and indirect costs, was estimated at $\$ 34.3$ billion in $2008 .^{5}$ Direct cost of AF-related strokes was estimated to be approximately $\$ 8$ billion. ${ }^{6}$

Clinical guidelines recommend the use of oral anticoagulants for long-term stroke prevention in AF patients who are at intermediate to high risk of stroke (i.e., $\mathrm{CHADS}_{2}$ score > 1), assuming patients are not at high bleeding risk. ${ }^{7,8}$ However, studies have consistently reported that many patients who were eligible for oral anticoagulation did not receive warfarin. Zimetbaum et al. $(2010)^{9}$ report that only $42.1 \%$ of patients with a $\mathrm{CHADS}_{2}$ score $\geq 3$ received warfarin therapy, and according to a systematic literature review, less than $60 \%$ of patients with high stroke risk are on oral anticoagulant (OAC) 
therapy. ${ }^{10}$ Contributing further to AF morbidity and mortality is poor adherence with warfarin therapy and high discontinuation rates among those who received prescriptions. ${ }^{11,12}$ According to a recent study of 4,188 Kaiser Permanente (U.S.) patients with AF, more than 1 in 4 patients (26\%) starting on warfarin discontinued therapy in the first year despite a low overall hemorrhage rate ( $2.3 \%$ of patients). ${ }^{13}$

Suboptimal utilization of OACs can lead to higher health care cost associated with strokes that would be prevented by effective anticoagulation therapy. As oral anticoagulation is also associated with an increased risk of bleeding, ${ }^{14}$ risk-benefit assessment is crucial in the anticoagulation treatment decision. Data on the economic impact of OAC underuse are limited. Based on decision-analytic modeling, Caro $(2004)^{6}$ estimated that optimizing INR (international normalized ratio) control among AF patients that received warfarin and increasing warfarin use in AF patients could result in a reduction of health care costs by $\$ 2.4$ billion per year. ${ }^{6}$

The objective of this study was to estimate the economic burden from the payers perspective associated with suboptimal utilization of warfarin therapy in a commercially insured AF population with moderate to high stroke risk without contraindications for warfarin treatment. This study utilized medical claims data to identify clinical events and costs and prescription claims data to assess warfarin use, which provides a more precise estimate of the economic implications of warfarin underuse and nonadherence than previous economic modeling.

\section{Methods}

\section{Study Design and Data Source}

This study was a retrospective, observational, quasi-experimental study using de-identified health care claims data from the Thomson Reuters' MarketScan Commercial Claims and Encounters and Medicare Supplemental and Coordination of Benefits databases from January 2003 to December 2007. These databases include individual-level enrollment and cost data across inpatient, outpatient, and prescription drug services from 45 large employers, health plans, and government and public organizations. The MarketScan database has been used in published research conducted in AF patients previously., ${ }^{9,15,16}$

\section{Study Population}

The study sample consisted of adult incident AF patients who would be candidates for OAC. Specifically, AF was identified based on the presence of at least 2 medical claims with primary or secondary AF diagnoses (using the International Classification of Diseases, Ninth Revision, Clinical Modification [ICD-9-CM] diagnosis code 427.31) separated by at least 30 days and not more than 12 months, of which at least 1 claim was in the outpatient setting. The date of the first AF medical claim was defined as the AF index date. Patients were required to have been continuously enrolled in the health plan for at least 12 months prior to the AF index date and at least 2 months after the $\mathrm{AF}$ index date to be eligible for inclusion.

Eligible patients were required to have moderate to high stroke risk as assessed by a $\mathrm{CHADS}_{2}$ score $\geq 2^{17}$ and did not have high bleed risk as assessed by an ATRIA bleeding risk score $<5,{ }^{18}$ the HEMORR 2 HAGES score $<4,{ }^{19}$ or the HAS-BLED score $<3 .{ }^{20}$ ICD-9-CM codes associated with inpatient and outpatient medical claims in the 12-month pre-index period were used to determine baseline stroke and bleeding risk scores. Patients with ICD-9-CM codes corresponding to contraindications to warfarin, according to the package insert for Coumadin, as well as those presenting with valvular and transient AF were excluded (see Appendices). Patients with warfarin prescription claims or prothrombin/INR (PT/INR) claims in the 12 months prior to AF index date were also excluded.

\section{Key Variables of Interest}

Warfarin exposure during the 18-month follow-up period after the AF index date was identified by the presence of outpatient prescription claims as well as the timing of PT/INR claims. Consistent with the algorithm validated by Go et al. (2003), ${ }^{21}$ patients were considered to be continually on warfarin when the gaps between 2 prescription claims were $\leq 60$ days apart. When the gaps between 2 prescription claims were $>60$ days apart, patients were considered to be continually on warfarin if there was a PT/INR claim every 42 days. If there was no PT/ INR claim every 42 days, then patients were considered off warfarin from the thirty-first day after the end of the first prescription days of supply until the start of the next prescription fill date. The level of warfarin exposure for patients who had at least 1 warfarin prescription claim was assessed using proportion of days covered (PDC), calculated as the total days of supply associated with warfarin prescription claims during the follow-up period (less any overlapping days of supply) divided by the length of follow-up after the AF index date. The level of warfarin exposure was classified as high (PDC >0.8) and low (PDC $\leq 0.8$ ), and patients who did not fill a warfarin prescription after the $\mathrm{AF}$ index date were considered to have a PDC $=0$ (no warfarin exposure). The use of the 0.8 cut point was based on conventions used in other conditions ${ }^{22}$ and based on the distribution of PDC values of warfarin users in our sample to ensure there was an adequate number of subjects in each PDC group.

Health care resource utilization and costs during the followup period for the no warfarin exposure group $(\mathrm{PDC}=0)$ were compared with those with high and low PDC groups. Inpatient hospitalizations, length of stay (LOS), emergency room (ER) visits, and outpatient office visits during the 18-month period after the AF index date was assessed. All cost variables were evaluated from the payer perspective and were based on 
The Costs of Warfarin Underuse and Nonadherence in Patients with Atrial Fibrillation: A Commercial Insurer Perspective

\begin{tabular}{l|l}
\hline \multicolumn{1}{c}{ TABLE 1) } \\
\multicolumn{1}{c}{$\begin{array}{c}\text { ICD-9-CM Codes for Stroke } \\
\text { and Bleed Outcomes }\end{array}$} \\
\hline Clinical Outcomes & \multicolumn{1}{c}{ ICD-9-CM Codes } \\
\hline Ischemic stroke & $43.00-01,433.10-11,433.20-21,433.30-31$, \\
& $434.00-01,434.10-11,434.90-91$, \\
& 436 (acute, ill-defined cerebrovascular disease) \\
\hline Other thromboembolic & $444.0,444.1,444.21-22,444.81,444.89,557.0$, \\
events & $557.1,557.9$ \\
\hline Intracranial hemorrhage & $430,431,432.0,432.1,432.9$ \\
& Note: all 5th digits 0-9 are searched for using \\
& the following traumatic ICH codes: \\
& $852.0,852.2,852.4,853.0$ \\
\hline Gastrointestinal & $455.2,455.5,455.8,456.0,456.20,459.0,530.7$, \\
hemorrhage & $530.82,531.00-01,531.20-21,531.40-41$, \\
& $531.60-61,532.00-01,532.20-21,532.40-41$, \\
& $532.60-61,533.00-01,533.20-21,533.40-41$, \\
& $533.60-61,534.00-01,534.20-21,534.40-41$, \\
& $534.60-61,535.01,535.11,535.21,535.31$, \\
& $535.41,535.51,535.61,537.83,562.02,562.03$, \\
& $562.12,562.13,568.81,569.3,569.85,578.0$, \\
& $578.1,578.9$ \\
\hline Other bleeds & 423.0 (hemopericardium) \\
& 593.81 (vascular disorders of kidney) \\
\hline Transient cerebral & 599.7 (hematuria) \\
\hline Ischemia [112.] & 719.11 (hemarthrosis: including fifth digits: 0-9) \\
\hline Modification; ICH=intracranial hemorrhage. & 784.7 (epistaxis) \\
\hline & 784.8 (hemorrhage from throat; added \\
& December 4, 2000) \\
\hline & 486.3 (hemoptysis) \\
\hline
\end{tabular}

amounts paid by the insurer to the provider (i.e., paid claims). Inpatient cost included services rendered during a hospital stay. Outpatient cost included physician office visits, hospital outpatient visits, ER visits, outpatient laboratory tests, medical procedures, and radiological exams, excluding outpatient prescription drug cost. Medical cost was defined as the total cost of all inpatient and outpatient medical services. Prescription drug cost included all outpatient pharmacy claims. Due to the variable length of follow-up for eligible study patients, cost measures were expressed on a per-patient per-month basis.

Ischemic stroke or bleeding events during the post-index period were assessed in each PDC group based on the presence of medical or inpatient claims with either a primary or secondary diagnosis (Table 1). Since the occurrence of strokes or bleeds may have resulted in the initiation or termination of warfarin therapy, which would influence warfarin exposure PDC levels, we censored the warfarin PDC calculation to either the day prior to the occurrence of such an event or at the end of the 18-month follow-up period, whichever occurred first.

\section{Statistical Analyses}

Descriptive analyses were used to compare baseline characteristics among patients at different PDC levels. A series of mul- tivariate regressions were performed to examine the effect of warfarin exposure level on health care resource use and costs. Negative binomial regression was used to assess health care resource use variables and a generalized linear model (GLM) with gamma distribution and log link was used to assess cost outcomes based on the skewed distribution encountered in our data and previous modeling studies of cost. ${ }^{23}$ When the proportion of patients with zero costs was $>10 \%$, two-part modeling using a preliminary logistic regression to obtain probabilities of incurring cost given the independent variables in the model, followed by a GLM on the subset of the population that incurred cost, was performed.

Covariates in the regression models included patient demographics, insurance type, comorbidities, stroke $\left(\mathrm{CHADS}_{2}\right)$ and bleed (ATRIA) risk factors, and pre-index measures of the same economic outcome variable under evaluation. Pre-index health care resource use and costs were identified by medical claims in the 12-month period before the AF index date. Comorbidities from the Elixhauser Comorbidity Inde ${ }^{24}$ were identified based on ICD-9-CM codes associated with inpatient and outpatient claims during the 12-month pre-index period. Due to a high degree of multicollinearity between ATRIA, HEMORR ${ }_{2} \mathrm{HAGES}$, and HAS-BLED bleeding risk scores (Pearson correlation coefficient $=0.6$ ), we controlled for ATRIA bleeding risk score ${ }^{13}$ in the models because the ATRIA risk factors can be more reliably defined using medical claims compared with the other indices. To account for variable follow-up time and to control its effect on cost variance, weights were determined based on the months of enrollment in the study divided by the study period (18 months) for each observation. All data management and analyses were conducted using SAS version 9.3 (SAS Institute Inc., Cary, NC).

\section{Results}

\section{Population Characteristics and Warfarin Utilization}

The study sample consisted of 13,289 patients with a $\mathrm{CHADS}_{2}$ score $\geq 2$ and no obvious contraindication to warfarin (Figure $1)$. Nearly half of the patients $(n=6,253 ; 47 \%)$ did not receive warfarin, and only $21.5 \%(n=2,852)$ of patients had a $\mathrm{PDC}>0.80$. Patients who did not receive warfarin were more likely to be $\geq 75$ years of age, and they had a slightly higher $\mathrm{CHADS}_{2}$ score (mean score $=2.37$ vs. 2.33; $P<0.001$ ) partly due to the higher prevalence of congestive heart failure than those who received warfarin. Patients who did not receive warfarin also had slightly higher bleeding risk on average as assessed by all 3 bleeding risk indices. Of the patients who did receive warfarin, $65 \%$ did so within 30 days of the index diagnosis. The mean follow-up time for patients not exposed to warfarin was 14.2 months, significantly lower compared with 15.6 months $(P<0.001)$ for patients who received warfarin (Table 2.1). There was a significantly greater proportion of high PDC patients with a history of bleeding compared with low PDC patients $(P<0.05$; Table 2.2). 
The Costs of Warfarin Underuse and Nonadherence in Patients with Atrial Fibrillation: A Commercial Insurer Perspective

\section{FIGURE 1 Patient Selection Flowchart}

INCLUDED

EXCLUDED

AF patients in MarketScan database from January 2003 to December 2007.

$\mathrm{N}=717,329$

Subjects with more than $1 \mathrm{AF}$ diagnoses as either inpatient or outpatient claims, listed as a principal or secondary diagnosis from January 1, 2003, to June 30, 2006, and separated by at least 30 days but not by more than 12 months.

At least 1 diagnosis should be in the outpatient setting.

$\mathrm{N}=299,495$

Age of $\geq 18$ years at the time of first diagnosis of $A F$.

$\mathrm{N}=297,260$

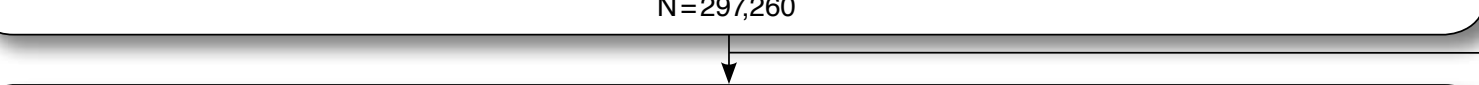

Continuous 12 months of enrollment prior to the first AF diagnosis date (prediagnosis period) and at least 2 months of continuous eligibility after the first $\mathrm{AF}$ diagnosis date.

$\mathrm{N}=105,734$

Absense of any diagnosis of valvular and/or transient AF:

Mitral stenosis, valvular repair or replacement, transient postoperative AF,

or concurrent ICD-9-CM codes for these conditions.

$$
\mathrm{N}=90,856
$$

No warfarin use during the 12-month period prior to the first AF diagnosis.

$\mathrm{N}=67,653$

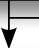

$\mathrm{CHADS}_{2}$ score $\geq 2$

$\mathrm{N}=25,744$

No deaths at first inpatient AF diagnosis.

$\mathrm{N}=25,741$
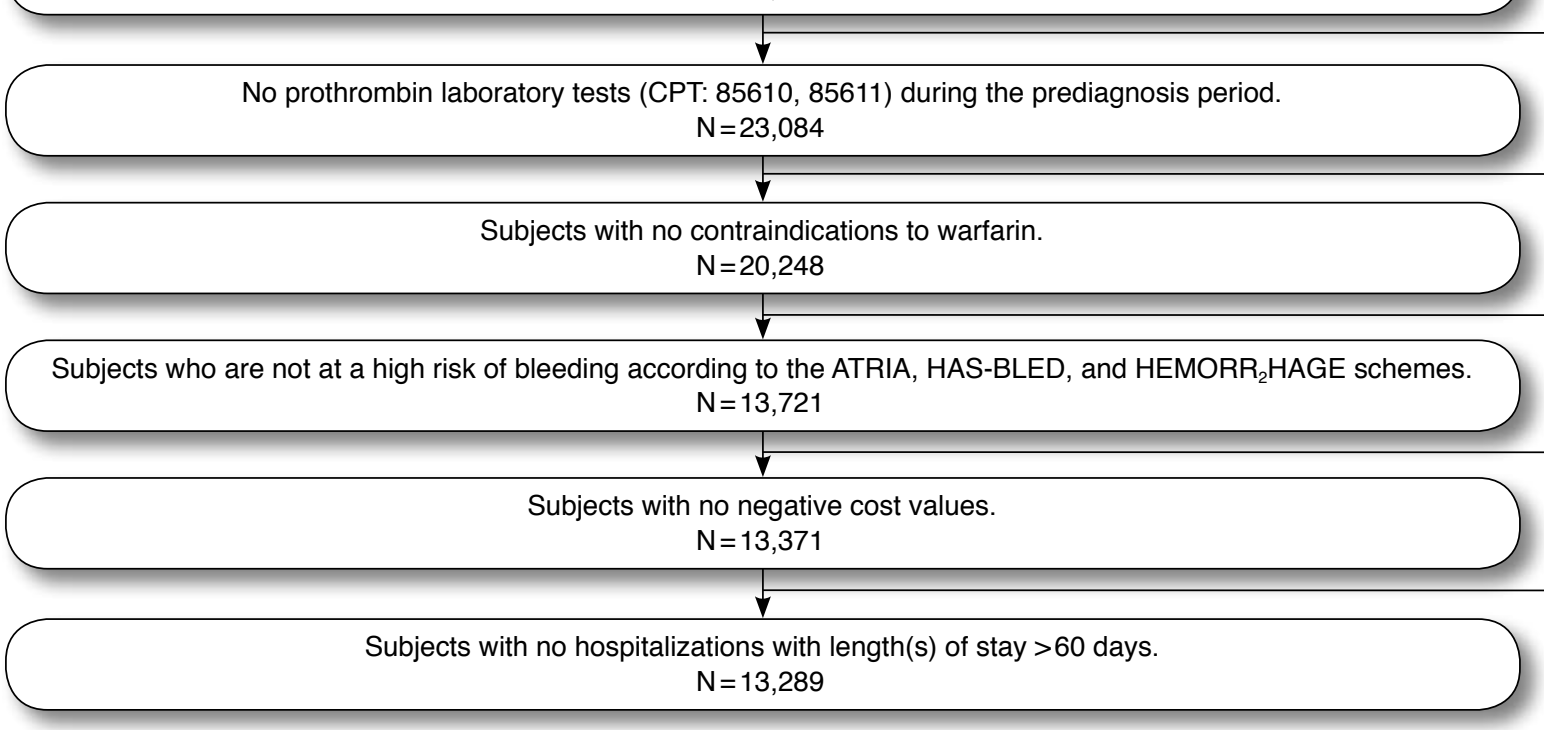

$\mathrm{N}=2,235$

$0.7 \%$

$\mathrm{N}=417,834$

$58 \%$

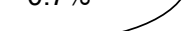

$\mathrm{N}=191,526$

$64 \%$

$N=14,878$

$14 \%$

$\mathrm{N}=23,203$

$26 \%$

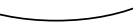

$\mathrm{N}=41,909$

$62 \%$

$\mathrm{N}=3$

$0.01 \%$

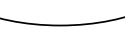

$\mathrm{N}=2,657$

$10 \%$

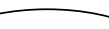

$\mathrm{N}=2,836$

$12 \%$

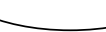

$\mathrm{N}=6,527$

$32 \%$
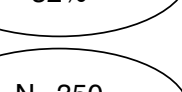

3\%

$\mathrm{N}=82$

$0.6 \%$ $\mathrm{N}=13,289$

$A F=$ atrial fibrillation; $C P T=$ Current Procedural Terminology; ICD-9-CM = International Classification of Diseases, Ninth Revision, Clinical Modification. 
TABLE 2.1 Summary Statistics: Demographics, Baseline Risk Factors, Comorbidities, and Insurance Type

\begin{tabular}{|c|c|c|c|c|}
\hline \multirow[t]{2}{*}{$\begin{array}{l}\text { Patient } \\
\text { Characteristics }\end{array}$} & \multicolumn{2}{|c|}{$\begin{array}{c}\text { Exposed to Warfarin } \\
(\text { PDC >0) } \\
\text { n (\%) }\end{array}$} & \multicolumn{2}{|c|}{$\begin{array}{c}\text { Unexposed to Warfarin } \\
(\text { PDC }=0) \\
\text { n }(\%)\end{array}$} \\
\hline & 7,036 & $(52.94)$ & 6,253 & $(47.06)$ \\
\hline \multicolumn{5}{|l|}{ Gender } \\
\hline Female & 3,612 & $(51.34)^{\mathrm{a}}$ & 3,426 & $(54.79)$ \\
\hline \multicolumn{5}{|l|}{ Age } \\
\hline$\geq 75$ years & 5,327 & $(75.71)^{\mathrm{a}}$ & 5,093 & $(81.45)$ \\
\hline \multicolumn{5}{|l|}{ Location $^{\mathrm{a}}$} \\
\hline Northeast & 607 & $(8.63)$ & 451 & $(7.21)$ \\
\hline North central & 2,545 & $(36.17)$ & 1,988 & $(31.79)$ \\
\hline South & 1,973 & $(28.04)$ & 1,740 & $(27.83)$ \\
\hline West & 1,885 & $(26.79)$ & 2,048 & $(32.75)$ \\
\hline Unknown & 26 & $(0.37)$ & 26 & $(0.42)$ \\
\hline \multicolumn{5}{|c|}{$\mathrm{CHADS}_{2}$ stroke risk factors } \\
\hline $\mathrm{CHF}$ & 1,473 & $(20.94)^{a}$ & 1,693 & $(27.08)$ \\
\hline Hypertension & 5,404 & $(76.81)^{\mathrm{a}}$ & 4,575 & $(73.16)$ \\
\hline Diabetes & 2,538 & $(36.07)^{\mathrm{a}}$ & 2,084 & $(33.33)$ \\
\hline Prior stroke or TIA & 782 & $(11.11)$ & 665 & $(10.63)$ \\
\hline \multicolumn{5}{|c|}{ ATRIA bleed risk factors } \\
\hline Anemia & 47 & $(0.67)^{\mathrm{b}}$ & 66 & $(1.1)$ \\
\hline Renal failure & 26 & $(0.51)$ & 32 & $(0.5)$ \\
\hline History of bleeding & 95 & $(1.35)$ & 90 & (1.4) \\
\hline \multicolumn{5}{|c|}{ Top 5 comorbidities (other than those included in CHADS 2 /ATRIA scales) } \\
\hline $\begin{array}{l}\text { Chronic pulmonary } \\
\text { disease }\end{array}$ & 1,612 & $(22.91)$ & 1,376 & $(22.01)$ \\
\hline Valvular disease & 1,463 & $(20.79)^{c}$ & 1,187 & $(18.98)$ \\
\hline $\begin{array}{l}\text { Peripheral vascular } \\
\text { disorders }\end{array}$ & 758 & $(10.77)$ & 720 & $(11.51)$ \\
\hline $\begin{array}{l}\text { Fluid electrolyte } \\
\text { disorders }\end{array}$ & 655 & $(9.31)^{a}$ & 701 & $(11.21)$ \\
\hline Hypothyroidism & 505 & $(7.18)^{\mathrm{a}}$ & 550 & $(8.80)$ \\
\hline \multicolumn{5}{|l|}{ Insurance type ${ }^{\mathrm{a}}$} \\
\hline $\begin{array}{l}\text { Comprehensive } \\
\text { insurance }\end{array}$ & 3,860 & $(55.47)$ & 3,105 & $(50.45)$ \\
\hline EPO/POS/CDHP & 257 & $(3.65)$ & 110 & $(1.8)$ \\
\hline $\mathrm{HMO}$ & 1,596 & $(22.93)$ & 1,743 & $(28.32)$ \\
\hline $\mathrm{PPO}$ & 1,323 & $(18.80)$ & 1,196 & (19.43) \\
\hline \multicolumn{5}{|l|}{ Warfarin initiation } \\
\hline $\begin{array}{l}\text { Within } 30 \text { days of } \\
\text { AF index diagnosis }\end{array}$ & 4,573 & $(64.99)$ & - & \\
\hline Risk scores & Mean (SD) & $\begin{array}{l}\text { Median } \\
\text { (Range) }\end{array}$ & Mean (SD) & $\begin{array}{l}\text { Median } \\
\text { (Range) }\end{array}$ \\
\hline $\mathrm{CHADS}_{2}$ & $2.33(0.60)^{\mathrm{a}}$ & $2(2-6)$ & $2.37(0.64)$ & $2(2-6)$ \\
\hline ATRIA & $2.33(0.93)^{\mathrm{a}}$ & $3(0-4)$ & $2.42(0.86)$ & $3(0-4)$ \\
\hline $\mathrm{HEMORR}_{2} \mathrm{HAGES}$ & $1.87(0.74)^{\mathrm{a}}$ & $2(0-3)$ & $1.92(0.73)$ & $2(0-3)$ \\
\hline $\begin{array}{l}\text { HAS-BLED } \\
\end{array}$ & $1.74(0.45)^{\mathrm{c}}$ & $2(0-2)$ & $1.72(0.46)$ & $2(0-2)$ \\
\hline \multicolumn{5}{|l|}{ Follow-up } \\
\hline $\begin{array}{l}\text { Months of follow-up, } \\
\text { mean (SD) }\end{array}$ & 15.6 & $(4.23)^{\mathrm{a}}$ & 14.2 & $(5.08)$ \\
\hline \multicolumn{5}{|c|}{$\begin{array}{l}\text { a } P<0.001 \text {, patients exposed to warfarin versus those who were not. } \\
b P<0.05 \text {, patients exposed to warfarin versus those who were not. } \\
c P<0.01 \text {, patients exposed to warfarin versus those who were not. } \\
A F=\text { atrial fibrillation; } C D H P=\text { consumer-directed health plan; } C H F=\text { congestive heart } \\
\text { failure; } E P O=\text { exclusive provider organizations; } H M O=\text { health maintenance organiza- } \\
\text { tion; } P D C=\text { proportion of days covered; } P O S=\text { point of service; } P P O=\text { preferred pro- } \\
\text { vider organization; } S D=\text { standard deviation; } T I A=\text { transient ischemic attack. }\end{array}$} \\
\hline
\end{tabular}

TABLE 2.2 Summary Statistics: Demographics, Baseline Risk Factors, Comorbidities, and Insurance Type: High and Low PDC

\begin{tabular}{|c|c|c|}
\hline Patient Characteristics & $\begin{array}{c}\text { High PDC }(>0.8) \\
\text { n (\%) }\end{array}$ & $\begin{array}{c}\text { Low PDC }(\leq 0.8) \\
n(\%)\end{array}$ \\
\hline & $\begin{array}{c}2,852 \\
\text { (21.46\% of total } \\
\text { population) }\end{array}$ & $\begin{array}{c}4,184 \\
\text { (31.48\% of total } \\
\text { population) }\end{array}$ \\
\hline \multicolumn{3}{|l|}{ Gender } \\
\hline Female & $(49.23)^{\mathrm{a}}$ & $(52.77)$ \\
\hline \multicolumn{3}{|l|}{ Age } \\
\hline$\geq 75$ years & $(72.72)^{\mathrm{b}}$ & $(77.75)$ \\
\hline \multicolumn{3}{|l|}{ Location $^{b}$} \\
\hline Northeast & $(7.36)$ & $(9.49)$ \\
\hline North central & $946 \quad(33.17)$ & $(38.22)$ \\
\hline South & $826 \quad(28.96)$ & $(27.41)$ \\
\hline West & $860 \quad(30.15)$ & $(24.50)$ \\
\hline Unknown & $(0.35)$ & $(0.38)$ \\
\hline \multicolumn{3}{|c|}{$\mathrm{CHADS}_{2}$ stroke risk factors } \\
\hline CHF & $(17.36)^{b}$ & $(23.37)$ \\
\hline Hypertension & $(77.88)^{\mathrm{c}}$ & (76.08) \\
\hline Diabetes & $(37.80)^{\mathrm{a}}$ & $(34.89)$ \\
\hline Prior stroke or TIA & $(11.61)$ & $(10.78)$ \\
\hline \multicolumn{3}{|l|}{ ATRIA bleed risk factors } \\
\hline Anemia & $(0.7)$ & $(0.65)$ \\
\hline Renal failure & $(0.53)$ & $(0.5)$ \\
\hline History of bleeding & $(1.72)^{\mathrm{c}}$ & $(1.1)$ \\
\hline
\end{tabular}

Top 5 comorbidities (other than those included in CHADS $_{2}$ /ATRIA scales) \begin{tabular}{l|ll|ll}
\hline Chronic pulmonary & 574 & $(20.13)^{\mathrm{b}}$ & 1,038 & $(24.81)$
\end{tabular}

disease

Valvular disease

Peripheral vascular

disorders

Fluid electrolyte disorders

Hypothyroidism

Insurance type $^{b}$

\begin{tabular}{l|rr|rr}
\hline Comprehensive insurance & 1,352 & $(47.79)$ & 2,508 & $(60.73)$ \\
\hline EPO/POS/CDHP & 98 & $(3.46)$ & 82 & $(1.98)$ \\
\hline $\mathrm{HMO}$ & 817 & $(28.88)$ & 779 & $(18.86)$ \\
\hline $\mathrm{PPO}$ & 562 & $(19.87)$ & 761 & $(18.43)$ \\
\hline
\end{tabular}

Follow-up

\begin{tabular}{l|ll|ll}
\hline Months of follow-up, & $15.6 \quad$ (4.27) & $15.6 \quad$ (4.19) \\
\hline
\end{tabular} mean (SD)

${ }^{a} P<0.01$, patients exposed to warfarin versus those who were not.

${ }^{b} P<0.001$, patients exposed to warfarin versus those who were not. ${ }^{c} P<0.05$, patients exposed to warfarin versus those who were not.

$C D H P=$ consumer-directed health plan; $C H F=$ congestive heart failure;

$E P O=$ exclusive provider organizations; $H M O=$ health maintenance organization; $P D C=$ proportion of days covered; $P O S=$ point of service; $P P O=$ preferred provider organization; $S D=$ standard deviation; TIA = transient ischemic attack

\section{Stroke and Bleed Rates}

Patients who received warfarin had significantly lower rates of ischemic stroke and transient ischemic attack (TIA) compared with those without warfarin exposure during the study followup, and the rates were consistently lowest among patients with high warfarin PDC (all $P$ values $<0.001$; Table 3 ). Considering 
The Costs of Warfarin Underuse and Nonadherence in Patients with Atrial Fibrillation: A Commercial Insurer Perspective

TABLE 3 Stroke and Bleed Rates Across Warfarin Exposure Level Groups During the Follow-Up Period

\begin{tabular}{|c|c|c|c|c|c|c|c|c|}
\hline \multirow[b]{2}{*}{ Group } & \multicolumn{2}{|c|}{$\begin{array}{l}\text { Unexposed to Warfarin } \\
\qquad \begin{array}{c}(\mathrm{PDC}=0) \\
\mathrm{n}=6,253\end{array}\end{array}$} & \multicolumn{3}{|c|}{$\begin{array}{c}\text { Low PDC }(\leq 0.8) \\
\mathbf{n}=4,184\end{array}$} & \multicolumn{3}{|c|}{$\begin{array}{c}\text { High PDC }(>0.8) \\
n=2,852\end{array}$} \\
\hline & $\mathbf{n}$ & $\begin{array}{l}\text { Rate per } 100 \\
\text { Person-Years }\end{array}$ & $\mathrm{n}$ & $\begin{array}{l}\text { Rate per } 100 \\
\text { Person-Years }\end{array}$ & $\begin{array}{l}P \text { Value } \\
\text { (Low vs. No } \\
\text { Exposure) }\end{array}$ & $\mathbf{n}$ & $\begin{array}{l}\text { Rate per } 100 \\
\text { Person-Years }\end{array}$ & $\begin{array}{c}P \text { Value } \\
\text { (High vs. No } \\
\text { Exposure) }\end{array}$ \\
\hline Ischemic stroke & 326 & 4.41 & 102 & 1.87 & $<0.001$ & 60 & 1.62 & $<0.001$ \\
\hline Transient ischemic attack & 131 & 1.77 & 39 & 0.72 & $<0.001$ & 17 & 0.46 & $<0.001$ \\
\hline Intracranial bleed & 40 & 0.54 & 32 & 0.59 & 0.449 & 24 & 0.65 & 0.285 \\
\hline Major GI bleed & 104 & 1.41 & 110 & 2.02 & 0.001 & 61 & 1.65 & 0.115 \\
\hline Other bleed & 16 & 0.22 & 13 & 0.29 & 0.602 & 13 & 0.35 & 0.116 \\
\hline Total bleeds & 158 & 1.73 & 152 & 2.79 & 0.001 & 97 & 2.62 & 0.012 \\
\hline
\end{tabular}

\section{TABLE 4 Unadjusted Mean (SD) Utilization Across Warfarin Exposure Groups During the} Pre- and Post-index Periods, Per Patient Per Month

\begin{tabular}{|c|c|c|c|c|c|c|c|c|c|c|c|}
\hline & \multicolumn{3}{|c|}{$\begin{array}{l}\text { Unexposed to Warfarin }(\mathrm{PDC}=0) \\
n=6,253\end{array}$} & \multicolumn{4}{|c|}{$\begin{array}{c}\text { Low PDC }(\leq 0.8) \\
n=4,184\end{array}$} & \multicolumn{4}{|c|}{$\begin{array}{c}\text { High PDC }(>0.8) \\
n=2,852\end{array}$} \\
\hline & $\begin{array}{l}\text { Mean } \\
\text { Pre-index } \\
\text { Visits } \\
\text { (SD) }\end{array}$ & $\begin{array}{c}\text { Mean } \\
\text { Post-index } \\
\text { Visits } \\
\text { (SD) }\end{array}$ & $\begin{array}{l}\text { Difference } \\
\text { in Post- and } \\
\text { Pre-index } \\
\text { Utilization }\end{array}$ & $\begin{array}{l}\text { Mean } \\
\text { Pre-index } \\
\text { Visits } \\
\text { (SD) }\end{array}$ & $\begin{array}{c}\text { Mean } \\
\text { Post-index } \\
\text { Visits } \\
\text { (SD) }\end{array}$ & $\begin{array}{c}\text { Difference } \\
\text { in Post- and } \\
\text { Pre-index } \\
\text { Utilization }\end{array}$ & $\begin{array}{c}P \text { Value } \\
\text { (Compared } \\
\text { with No } \\
\text { Exposure) }\end{array}$ & $\begin{array}{c}\text { Mean } \\
\text { Pre-index } \\
\text { Visits (SD) }\end{array}$ & $\begin{array}{c}\text { Mean } \\
\text { Post-index } \\
\text { Visits (SD) }\end{array}$ & $\begin{array}{l}\text { Difference } \\
\text { in Post- and } \\
\text { Pre-index } \\
\text { Utilization }\end{array}$ & $\begin{array}{c}P \text { Value } \\
\text { (Compared } \\
\text { with No } \\
\text { Exposure) }\end{array}$ \\
\hline $\begin{array}{l}\text { Inpatient } \\
\text { encounters }\end{array}$ & $\begin{array}{c}0.064 \\
(0.075)\end{array}$ & $\begin{array}{c}0.088 \\
(0.124)\end{array}$ & 0.024 & $\begin{array}{c}0.073 \\
(0.075)\end{array}$ & $\begin{array}{c}0.091 \\
(0.113)\end{array}$ & 0.018 & 0.352 & $\begin{array}{c}0.071 \\
(0.070)\end{array}$ & $\begin{array}{c}0.063 \\
(0.088)\end{array}$ & -0.008 & $<0.001$ \\
\hline $\begin{array}{l}\text { Outpatient } \\
\text { encounters }\end{array}$ & $\begin{array}{c}1.449 \\
(0.958)\end{array}$ & $\begin{array}{l}2.195 \\
(1.393)\end{array}$ & 0.746 & $\begin{array}{c}1.421 \\
(0.942)\end{array}$ & $\begin{array}{l}2.595 \\
(1.379) \\
\end{array}$ & 1.174 & $<0.001$ & $\begin{array}{c}1.334 \\
(0.854) \\
\end{array}$ & $\begin{array}{c}2.816 \\
(1.364)\end{array}$ & 1.482 & $<0.001$ \\
\hline $\begin{array}{l}\text { ER } \\
\text { encounters }\end{array}$ & $\begin{array}{c}0.078 \\
(0.117) \\
\end{array}$ & $\begin{array}{c}0.121 \\
(0.179) \\
\end{array}$ & 0.043 & $\begin{array}{c}0.075 \\
(0.116) \\
\end{array}$ & $\begin{array}{c}0.128 \\
(0.181) \\
\end{array}$ & 0.053 & $<0.001$ & $\begin{array}{c}0.060 \\
(0.096) \\
\end{array}$ & $\begin{array}{c}0.093 \\
(0.174) \\
\end{array}$ & 0.033 & 0.004 \\
\hline LOS & $\begin{array}{c}0.298 \\
(0.688) \\
\end{array}$ & $\begin{array}{c}0.393 \\
(0.953) \\
\end{array}$ & 0.095 & $\begin{array}{c}0.276 \\
(0.607) \\
\end{array}$ & $\begin{array}{c}0.381 \\
(0.813) \\
\end{array}$ & 0.105 & 0.673 & $\begin{array}{c}0.223 \\
(0.370) \\
\end{array}$ & $\begin{array}{c}0.212 \\
(0.554) \\
\end{array}$ & -0.011 & $<0.001$ \\
\hline
\end{tabular}

$E R=$ emergency room; $L O S=$ length of stay; $P D C=$ proportion of days covered; $S D=$ standard deviation.

all types of bleeding events (major, minor, and other), patients who received warfarin had a significantly higher bleeding rate than patients who did not receive warfarin $(P<0.05)$. There was no significant difference in the rate of intracranial hemorrhage between patients who received warfarin and patients who did not receive warfarin. However, the major gastrointestinal (GI) hemorrhage rate was significantly higher among patients with warfarin $\mathrm{PDC} \leq 0.80$ than patients who did not receive warfarin. The risk of major GI bleeding was similar between patients with high warfarin PDC and patients who did not receive warfarin.

\section{Health Care Resource Utilization}

Table 4 summarizes unadjusted health care service utilization differences between the pre-index and post-index follow-up period. Differences in post- and pre-index hospitalizations $(-0.008$ vs. $0.024 ; P<0.001)$ and $\operatorname{LOS}(-0.011$ vs. 0.095; $P<0.001)$ were significantly lower for the high warfarin PDC group compared with no warfarin. Increases in post-index outpatient encounters (from pre-index) were significantly greater for low $(1.174$ vs. $0.746 ; P<0.001)$ and high warfarin $(1.482$ vs. 0.746; $P<0.001)$ PDC groups compared with the no exposure group. The increase in number of ER encounters between the pre- and post-index periods was significantly lower in the high PDC group (0.033 vs. $0.043 ; P=0.004)$ and higher in the low PDC group (0.053 vs. 0.043; $P<0.001)$ compared with the group with no warfarin exposure.

After controlling for covariates and baseline utilization, the high warfarin PDC group was associated with $27 \%$ fewer inpatient encounters $(P<0.001), 16 \%$ fewer ER visits $(P=0.019)$, and nearly a $40 \%$ reduction in the number of hospital days $(P<0.001)$ compared with patients who did not receive warfarin (Figure 2). In contrast, inpatient and ER services utilization were not significantly different between patients with low warfarin PDC and patients who did not receive warfarin. Patients who received warfarin, regardless of PDC level, had 


\section{TABLE 5 Unadjusted Mean (SD) Costs Across Warfarin Exposure Groups During the} Pre- and Post-index Periods, Per Patient Per Month (\$US)

\begin{tabular}{|c|c|c|c|c|c|c|c|c|c|c|c|}
\hline & \multicolumn{3}{|c|}{$\begin{array}{l}\text { Unexposed to Warfarin } \\
(P D C=0) n=6,253\end{array}$} & \multicolumn{4}{|c|}{$\begin{array}{c}\text { Low PDC }(\leq 0.8) \\
n=4,184\end{array}$} & \multicolumn{4}{|c|}{$\begin{array}{c}\text { High PDC }(>0.8) \\
n=2,852\end{array}$} \\
\hline & $\begin{array}{c}\text { Mean } \\
\text { Pre-index } \\
\text { Cost } \\
\text { (SD) }\end{array}$ & $\begin{array}{c}\text { Mean } \\
\text { Post-index } \\
\text { Cost } \\
\text { (SD) }\end{array}$ & $\begin{array}{l}\text { Difference } \\
\text { in Post- } \\
\text { and Pre- } \\
\text { index } \\
\text { Utilization }\end{array}$ & $\begin{array}{c}\text { Mean } \\
\text { Pre-index } \\
\text { Cost } \\
\text { (SD) }\end{array}$ & \begin{tabular}{|} 
Mean \\
Post-index \\
Cost \\
(SD)
\end{tabular} & $\begin{array}{c}\text { Difference } \\
\text { in Post- } \\
\text { and Pre- } \\
\text { index } \\
\text { Utilization }\end{array}$ & $\mid \begin{array}{c}P \text { Value } \\
\text { (Compared } \\
\text { with No } \\
\text { Exposure) }\end{array}$ & $\begin{array}{c}\text { Mean } \\
\text { Pre-index } \\
\text { Cost } \\
\text { (SD) }\end{array}$ & \begin{tabular}{|c|} 
Mean \\
Post-index \\
Cost \\
(SD)
\end{tabular} & \begin{tabular}{|} 
Difference \\
in Post- \\
and Pre- \\
index \\
Utilization
\end{tabular} & $\begin{array}{c}P \text { Value } \\
\text { (Compared } \\
\text { with No } \\
\text { Exposure) }\end{array}$ \\
\hline $\begin{array}{l}\text { All-cause inpatient } \\
\text { costs }\end{array}$ & $\begin{array}{l}180.69 \\
(732.45) \\
\end{array}$ & $\begin{array}{c}314.15 \\
(2,491.19)\end{array}$ & 133.46 & $\begin{array}{l}184.86 \\
(673.31) \\
\end{array}$ & $\begin{array}{c}273.57 \\
(1,612.26) \\
\end{array}$ & 104.46 & 0.477 & $\begin{array}{l}213.46 \\
(611.45) \\
\end{array}$ & $\begin{array}{c}228.91 \\
(1,099.59) \\
\end{array}$ & 15.45 & 0.002 \\
\hline Stroke-related & $\begin{array}{c}8.81 \\
(113.12) \\
\end{array}$ & $\begin{array}{c}29.01 \\
(629.13) \\
\end{array}$ & 20.20 & $\begin{array}{c}11.98 \\
(146.28) \\
\end{array}$ & $\begin{array}{c}6.31 \\
(90.73) \\
\end{array}$ & -5.67 & 0.002 & $\begin{array}{c}19.77 \\
(151.77)\end{array}$ & $\begin{array}{c}8.63 \\
(181.45) \\
\end{array}$ & -11.14 & 0.0006 \\
\hline Bleed-related & $\begin{array}{c}1.67 \\
(30.94)\end{array}$ & $\begin{array}{c}9.44 \\
(267.89)\end{array}$ & 7.77 & $\begin{array}{c}1.25 \\
(36.27)\end{array}$ & $\begin{array}{c}10.25 \\
(272.14)\end{array}$ & 9 & 0.8 & $\begin{array}{c}0.24 \\
(11.44)\end{array}$ & $\begin{array}{c}5.66 \\
(62.94)\end{array}$ & 5.42 & 0.5 \\
\hline $\begin{array}{l}\text { All-cause outpatient } \\
\text { costs }\end{array}$ & $\begin{array}{c}122.63 \\
(315.26) \\
\end{array}$ & $\begin{array}{l}309.94 \\
(717.04) \\
\end{array}$ & 187.31 & $\begin{array}{c}123.2 \\
(664.83) \\
\end{array}$ & $\begin{array}{l}278.15 \\
(643.67) \\
\end{array}$ & 154.94 & 0.028 & $\begin{array}{c}120.24 \\
(333.58) \\
\end{array}$ & $\begin{array}{c}242.18 \\
(558.63)\end{array}$ & 121.94 & $<0.001$ \\
\hline Stroke-related & $\begin{array}{c}2.07 \\
(22.25)\end{array}$ & $\begin{array}{l}10.07 \\
(83.31)\end{array}$ & 8.00 & $\begin{array}{c}2.34 \\
(17.09)\end{array}$ & $\begin{array}{c}8.66 \\
(109.07)\end{array}$ & 6.32 & 0.4 & $\begin{array}{c}2.17 \\
(15.18)\end{array}$ & $\begin{array}{c}3.53 \\
(26.84)\end{array}$ & 1.36 & $<0.0001$ \\
\hline Bleed-related & $\begin{array}{c}0.26 \\
(5.68)\end{array}$ & $\begin{array}{c}4.91 \\
(50.78) \\
\end{array}$ & 4.65 & $\begin{array}{c}0.14 \\
(3.35)\end{array}$ & $\begin{array}{c}4.22 \\
(44.88) \\
\end{array}$ & 4.08 & 0.5 & $\begin{array}{c}0.26 \\
(3.93)\end{array}$ & $\begin{array}{c}3.06 \\
(21.52)\end{array}$ & 2.8 & 0.01 \\
\hline $\begin{array}{l}\text { All-cause prescription } \\
\text { drug costs }\end{array}$ & $\begin{array}{l}156.03 \\
(215.14)\end{array}$ & $\begin{array}{l}174.96 \\
(242.15)\end{array}$ & 18.93 & $\begin{array}{l}181.96 \\
(198.51)\end{array}$ & $\begin{array}{l}223.04 \\
(215.77)\end{array}$ & 41.09 & $<0.001$ & $\begin{array}{l}172.58 \\
(207.89)\end{array}$ & $\begin{array}{l}222.54 \\
(216.82)\end{array}$ & 49.96 & $<0.001$ \\
\hline Total costs & $\begin{array}{c}459.35 \\
(906.43)\end{array}$ & $\begin{array}{c}799.06 \\
(2,746.66)\end{array}$ & 339.71 & $\begin{array}{c}490.02 \\
(1,138.29)\end{array}$ & $\begin{array}{c}790.52 \\
(2,056.73)\end{array}$ & 300.49 & 0.392 & $\begin{array}{l}506.28 \\
(826.48)\end{array}$ & $\begin{array}{c}693.63 \\
(1,459.91)\end{array}$ & 187.35 & $<0.001$ \\
\hline
\end{tabular}

$P D C=$ proportion of days covered; $S D=$ standard deviation.

FIGURE 2 Adjusted Utilization Ratios (95\% Cl): Post-index Resource Utilization Among Low $(\leq 0.8)$ and High $(>0.8)$ PDC Warfarin Users Compared with Patients Who Did Not Receive Warfarin

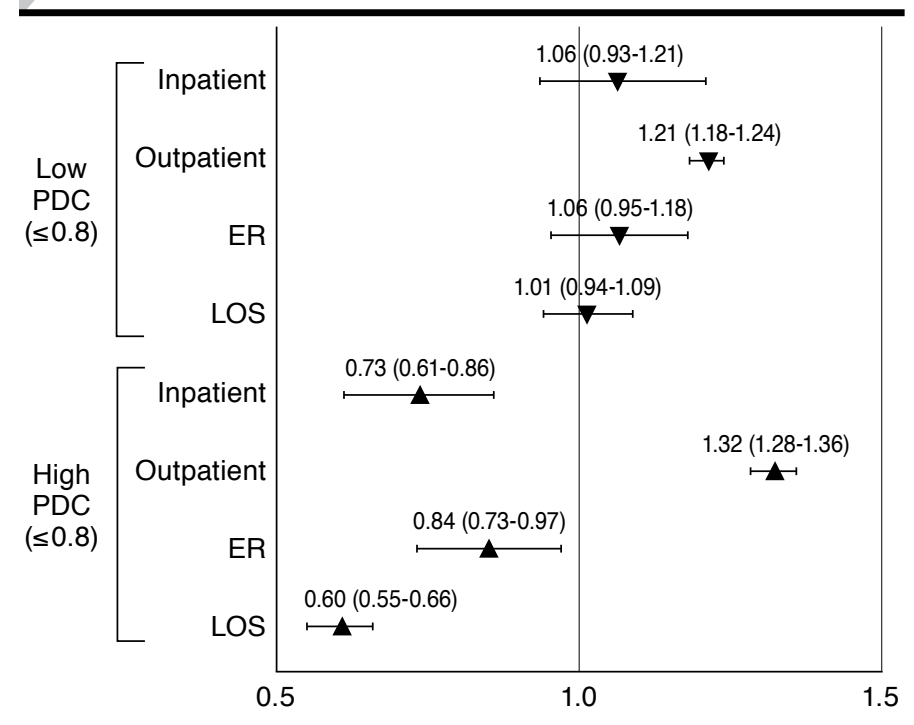

Adjusted Utilization Ratio (95\% Cl)

Favors warfarin use Favors no warfarin use

$C I=$ confidence interval; ER=emergency room; $L O S=$ length of stay; $P D C=$ proportion of days covered. significantly more outpatient visits in the post-index period than patients who did not receive warfarin. Patients with low and high PDCs had 21\% and 32\% greater outpatient visits, respectively, during follow-up compared with those who did not receive warfarin $(P<0.001$ for both $)$

\section{Health Care Costs}

Increases in pre- versus post-index period inpatient, outpatient, and total cost were significantly lower for the high PDC group, compared with the group with no warfarin exposure (15.45 vs. $133.46, P=0.002 ; 121.94$ vs. $187.31, P<0.001 ; 187.35$ vs. 339.71, $P<0.001$, respectively). Patients with high PDC showed a decrease in stroke-related inpatient cost in the postindex period (-\$11.14), significantly lower than patients with no warfarin use who showed an increase in these costs in the post-index period $(\$ 20.20 ; P=0.0006)$. Additionally, outpatient stroke-related cost increases from the pre-index period were significantly lower for the high warfarin PDC group compared with patients with no warfarin exposure (1.36 vs. 8.00, $P<0.0001$; Table 5).

Adjusted mean pharmacy cost was significantly higher for both patients with low (adjusted cost ratio $=1.08 ; P<0.001$ ) and high PDCs (adjusted cost ratio $=1.16 ; P<0.001$ ) than patients who did not receive warfarin (Table 6). After controlling for covariates and pre-index health care cost, adjusted mean allcause inpatient (adjusted cost ratio $=0.88 ; P<0.001$ ), all-cause outpatient (adjusted cost ratio $=0.73 ; P<0.001$ ), and all-cause 
The Costs of Warfarin Underuse and Nonadherence in Patients with Atrial Fibrillation: A Commercial Insurer Perspective

TABLE 6 Generalized Linear Model Estimation of Adjusted Mean Monthly Cost During Study Follow-up Period ${ }^{a}$

\begin{tabular}{|c|c|c|c|c|c|c|c|}
\hline \multirow[b]{2}{*}{ Parameter } & \multirow{2}{*}{$\begin{array}{c}\text { No Warfarin } \\
\text { Exposure } \\
\text { Mean Adjusted } \\
\text { Cost Estimate } \\
\text { \$ (SD) }\end{array}$} & \multicolumn{3}{|c|}{ Low PDC Warfarin Exposure } & \multicolumn{3}{|c|}{ High PDC Warfarin Exposure } \\
\hline & & $\begin{array}{c}\text { Exponentiated } \\
\text { Coefficient/Cost } \\
\text { Ratio (SD) }\end{array}$ & \multirow{2}{*}{$\frac{95 \% \text { CI }}{0.85-0.95}$} & $\begin{array}{c}\text { Mean Adjusted } \\
\text { Cost Estimate } \\
\text { \$(SD) }\end{array}$ & $\begin{array}{c}\text { Exponentiated } \\
\text { Coefficient/Cost } \\
\text { Ratio (SD) }\end{array}$ & \multirow{2}{*}{$\begin{array}{c}95 \% \text { CI } \\
0.82-0.94\end{array}$} & $\begin{array}{c}\text { Mean Adjusted } \\
\text { Cost Estimate } \\
\text { \$(SD) }\end{array}$ \\
\hline $\begin{array}{l}\text { All-cause inpatient cost } \\
\text { (in cases where } \\
\text { inpatient admission } \\
\text { cost was incurred) }\end{array}$ & $870.79 \quad(1.12)$ & $0.90^{\mathrm{b}}(1.03)$ & & 781.88 (1.12) & $0.88^{\mathrm{b}}(1.03)$ & & $763.34(1.12)$ \\
\hline $\begin{array}{l}\text { All-cause outpatient } \\
\text { cost }\end{array}$ & 747.32 (1.11) & $0.96 \quad(1.02)$ & $0.92-1.01$ & $717.16 \quad(1.11)$ & $0.73^{b}(1.03)$ & $0.69-0.77$ & $542.61 \quad(1.12)$ \\
\hline All-cause medical cost & $1,304.53 \quad(1.12)$ & $0.97 \quad(1.02)$ & $0.93-1.02$ & $1,269.65 \quad(1.12)$ & $0.72^{\mathrm{b}} \quad(1.03)$ & $0.68-0.76$ & $937.30 \quad(0.72)$ \\
\hline $\begin{array}{l}\text { Stroke-related medical } \\
\text { cost (in cases where } \\
\text { stroke-related medical } \\
\text { cost was incurred) }\end{array}$ & $140.56 \quad(1.38)$ & $0.52^{b}(1.08)$ & $0.45-0.61$ & $73.16 \quad(1.38)$ & $0.40^{\mathrm{b}}(1.10)$ & $0.33-0.48$ & $56.22(1.39)$ \\
\hline $\begin{array}{l}\text { Bleed-related medical } \\
\text { cost (in cases where } \\
\text { bleed-related medical } \\
\text { cost was incurred) } \\
\end{array}$ & $33.61 \quad(1.35)$ & $1.05 \quad(1.09)$ & $0.89-1.24$ & $35.39(1.35)$ & $0.78^{c} \quad(1.11)$ & $0.65-0.95$ & $26.37(1.36)$ \\
\hline Rx drug cost & $183.46 \quad(1.07)$ & $1.08^{\mathrm{b}}(1.02)$ & $1.05-1.12$ & $199.02(1.08)$ & $1.16^{\mathrm{b}}(1.02)$ & $1.12-1.20$ & $213.24 \quad(1.08)$ \\
\hline Total cost & $1,344.13 \quad(1.09)$ & $1.03 \quad(1.02)$ & $0.99-1.07$ & $1,384.37 \quad(1.09)$ & $0.87^{b} \quad(1.02)$ & $0.83-0.90$ & $1,164.56 \quad(1.09)$ \\
\hline $\begin{array}{l}{ }^{a} \text { All models were adjusted } \\
{ }^{b} P<0.001 \text {, patients with } l \\
{ }^{c} P<0.05 \text {, patients with lov } \\
C I=\text { confidence interval; } P\end{array}$ & $\begin{array}{l}\text { or previous resource } \\
\text { w or high warfarin e } \\
\text { or high warfarin ex } \\
C=\text { proportion of } d c\end{array}$ & $\begin{array}{l}\text { use, gender, age, regi } \\
\text { xposure versus those } \\
\text { posure versus those w } \\
\text { lys covered; Rx = pres }\end{array}$ & $\begin{array}{l}\text { insurance ca } \\
\text { n no exposur } \\
\text { no exposure. } \\
\text { tion; } S D=\text { stc }\end{array}$ & r, ATRIA score, CHA & $A D S_{2}$ score, and como & lities. & \\
\hline
\end{tabular}

medical (adjusted cost ratio $=0.72 ; P<0.001$ ) costs were significantly lower in patients with high warfarin PDC than patients who did not receive warfarin. Stroke-related medical cost was $60 \%$ lower (adjusted cost ratio $=0.40 ; P<0.001$ ) and bleed-related medical cost was 22\% lower (adjusted cost ratio $=0.78 ; P<0.05$ ) for patients with high warfarin PDC compared with those with no warfarin exposure. Patients with low warfarin PDC had significantly lower mean adjusted all-cause inpatient cost (adjusted cost ratio $=0.90 ; P<0.001$ ) but similar all-cause outpatient cost (adjusted cost ratio $=0.96$; $P=0.09$ ) and all-cause medical cost (adjusted cost ratio $=0.97$; $P=0.27)$ compared with patients who did not receive warfarin. Stroke-related medical cost was $48 \%$ lower for patients with low warfarin PDC compared with patients with no warfarin exposure. Overall, total health care costs were 13\% lower for patients with high PDC $(P<0.001)$ but similar for patients with low PDC compared with patients who did not receive warfarin.

\section{Discussion}

Our study found that only 53\% of patients with moderate to high risk of stroke and not at high bleeding risk received warfarin therapy. Even among patients who received warfarin, only $40 \%$ were adherent to warfarin with a PDC $>0.80$. Previous studies using administrative claims and patient registries have found warfarin underuse to be a prevalent problem in $\mathrm{AF}$ patients $^{9,10}$ and that maintaining optimal INR control ${ }^{25}$ and minimizing unintended discontinuation of warfarin therapy to be a significant challenge among patients who had received warfarin therapy. ${ }^{13,26}$ Our study represents one of the first attempts to estimate the economic burden associated with warfarin underuse and nonadherence. Although AF patients who received warfarin had higher pharmacy cost and incurred more outpatient visits, adherence to OAC therapy resulted in fewer inpatient admissions and ER visits, leading to significant reductions in total health care cost. Greater adherence as measured by PDC, compared with no use, clearly shows beneficial economic results in terms of lower total, inpatient, and outpatient costs during follow-up. Ischemic stroke rates for the low and high warfarin exposure groups were significantly lower than the no warfarin exposure group. However, there was not a significant difference in the ischemic strokes rates between the high and low warfarin PDC groups. While there was a greater rate of GI bleeds among the low PDC group compared with those not taking warfarin $(P=0.001)$, we did not find a significant difference between the high PDC and no warfarin group. This observation may be explained through a widely established association between poor adherence to warfarin and unstable INR, which has been shown to increase bleeding risk. ${ }^{13,20}$ This relationship between poor adherence and bleeding risk may also explain the significantly greater number of observed ER encounters for the low PDC group. 
The findings of our study should be viewed in light of several limitations. First, although the study used a large nationally representative database of patients recently diagnosed with AF, it may be subject to selection bias. The MarketScan database under-represents the U.S. Medicare population, a dominant patient segment in AF. Additionally, in working with large sample sizes, observed differences may be statistically significant but clinically irrelevant. For example, our observed baseline difference in the $\mathrm{CHADS}_{2}$ score (mean score $=2.33$ vs. 2.37; $P<0.001$ ) between patients that used and did not use warfarin may not translate to notable clinical difference in stroke risk. Patients were required to have at least 2 months of continuous eligibility after the AF index date. Patients who died within 2 months of the index AF diagnosis were not included in the study. By excluding patients who may have died from stroke or intracranial bleeding, the study can potentially over- or underestimate the cost of warfarin underutilization. Second, as with other retrospective claims data analysis, adherence in this study was measured by prescription claim records. In the absence of additional clinical information, we were unable to determine how well patients follow the instructions given by their health care providers and if discontinuation of warfarin therapy is medically necessary. Third, we defined eligibility of OAC therapy using $\mathrm{CHADS}_{2}$ and published bleeding risk algorithms. Although the $\mathrm{CHADS}_{2}$ score has been available and validated for stroke risk stratification, it remains unclear to what extent it has been routinely used in clinical practice to guide anticoagulation decisions. Current treatment guidelines for anticoagulation do not specify a standard instrument that should be used for bleeding risk assessment. ${ }^{8}$ Limited data exist on how well these bleeding risk scores can predict bleeding in clinical practice and to facilitate risk-benefit assessment of OAC treatment decisions. More data on the validity of these scores in clinical practice are needed.

This study identifies a significant economic and clinical burden driven by previously established warfarin underutilization among patients with AF. These findings reveal a missed opportunity to reduce costs by avoiding strokes that can be effectively prevented by OAC therapy. The results underscore the need for actions by health insurers and policy makers to develop programs to overcome these challenges in prescribing OAC therapy.

\section{Conclusion}

The economic implications of underutilizing warfarin in nearly half of the commercially insured nonvalvular AF population are significant. Adherence in terms of greater warfarin exposure was shown to reduce health care resource use as well as overall health care cost. Given the chronic nature of AF, improving adherence is critical to attaining the clinical and economic benefits of OAC therapy.

\section{Authors}

JULIAN P. CASCIANO, BS, is CEO and President, and ZENOBIA J. DOTIWALA, MS, is Senior Analyst, eMAX Health Systems, LLC, White Plains, New York. BRADLEY C. MARTIN, PharmD, PhD, is Professor and Director, Division of Pharmaceutical Evaluation and Policy, College of Pharmacy, University of Arkansas for Medical Sciences, Little Rock, Arkansas, and WINGHAN JACQUELINE KWONG, PharmD, PhD, is Senior Director, Health Economics \& Outcomes Research, Daiichi Sankyo, Inc., Parsippany, New Jersey.

AUTHOR CORRESPONDENCE: Julian P. Casciano, BS, eMAX Health Systems, LLC, 445 Hamilton Ave., White Plains, NY 10601. Tel.: 914.304.8128; Fax: 914.206.4959;

E-mail: juliancasciano@emaxhealth.net.

\section{DISCLOSURES}

This study was funded by Daiichi Sankyo, Inc. Casciano and Dotiwala are employed at eMAX Health Systems, LLC, which received research funding from Daiichi Sankyo to conduct this analysis. eMAX Health has also received funding from Pfizer, Inc., for other studies. Kwong is an employee of Daiichi Sankyo, Inc., and Martin is a consultant to eMAX Health and Daiichi Sankyo for studies related to the risks and benefits of oral anticoagulation. He is also a consultant to Bayer for cost studies in pulmonary hypertension.

Concept and design were performed by Casciano, Kwong, Dotiwala, and Martin. Data were collected by Martin, Casciano, Moore, and Dotiwala and interpreted by Dotiwala, Casciano, Martin, Kwong, and Szamreta. The manuscript was written by Casciano, Dotiwala, Kwong, and Martin and was revised by Casciano with the help of Dotiwala, Martin, and Kwong.

\section{ACKNOWLEDGEMENTS}

The authors wish to thank Elizabeth Szamreta, MPH, University of Medicine and Dentistry of New Jersey, for assistance with analysis and review, as well as Gary Moore, MS, University of Arkansas, Little Rock, for help in preparing the analytic extracts from the MarketScan database.

\section{REFERENCES}

1. Naccarelli GV, Varker H, Lin J, Schulman KL. Increasing prevalence of atrial fibrillation and flutter in the United States. Am J Cardiol. 2009;104(11):1534-39.

2. Camm AJ. Stroke prevention in atrial fibrillation-the unmet need and morbidity burden. European Cardiology. 2011;7(3):187-95. Available at: http:// www.touchcardiology.com/articles/stroke-prevention-atrial-fibrillationunmet-need-and-morbidity-burden. Accessed April 5, 2013.

3. Wolf PA, Abbott RD, Kannel WB. Atrial fibrillation as an independent risk factor for stroke: the Framingham Study. Stroke. 1991;22(8):983-88. Available at: http://stroke.ahajournals.org/content/22/8/983.full.pdf+html. Accessed April 5, 2013.

4. Coyne KS, Paramore C, Grandy S, Mercader M, Reynolds M, Zimetbaum P. Assessing the direct costs of treating nonvalvular atrial fibrillation in the United States. Value Health. 2006;9(5):348-56

5. Roger VL, Go AS, Lloyd-Jones DM, et al. Heart disease and stroke statistics-2012 update: a report from the American Heart Association. Circulation. 2012;125(1):e2-e220. Available at: http://circ.ahajournals.org/ content/125/1/e2. Accessed April 5, 2013 
6. Caro JJ. An economic model of stroke in atrial fibrillation: the cost of suboptimal oral anticoagulation. Am J Managed Care. 2004;10(14 Suppl):S451-S461. Available at: http://www.ajmc.com/publications/ supplement/2004/2004-12-voll0-n14Suppl/Dec04-1992pS451-S461/. Accessed April 5, 2013.

7. Camm AJ, Kirchhof P, Lip GYH, et al. Guidelines for the management of atrial fibrillation: the Task Force for the Management of Atrial Fibrillation of the European Society of Cardiology (ESC). Eur Heart J. 2010;31(19):2369429. Available at: http://eurheartj.oxfordjournals.org/content/31/19/2369. long. Accessed April 5, 2013.

8. You JJ, Singer DE, Howard PA, et al. Antithrombotic therapy for atrial fibrillation: Antithrombotic Therapy and Prevention of Thrombosis, 9th ed: American College of Chest Physicians Evidence-Based Clinical Practice Guidelines. Chest. 2012;141(2 Suppl):e531S-e575S. Available at: http://journal.publications.chestnet.org/article. aspx?articleid=1159549. Accessed April 5, 2013.

9. Zimetbaum PJ, Thosani A, Yu HT, et al. Are atrial fibrillation patients receiving warfarin in accordance with stroke risk? Am J Med. 2010;123(5):446-53. Available at: http://www.amjmed.com/article/S00029343(09)01114-0/fulltext. Accessed April 5, 2013.

10. Ogilvie IM, Newton N, Welner SA, Cowell W, Lip GY. Underuse of oral anticoagulants in atrial fibrillation: a systematic review. Am J Med. 2010;123(7):638-45. Available at: http://www.amjmed.com/article/S00029343(10)00254-8/fulltext. Accessed April 5, 2013.

11. Kimmel SE, Chen Z, Price M, et al. The influence of patient adherence on anticoagulation control with warfarin: results from the International Normalized Ratio Adherence and Genetics (IN-RANGE) Study. Arch Intern Med. 2007;167(3):229-35. Available at: http://archinte.jamanetwork.com/ article. aspx?articleid=411612. Accessed April 5, 2013.

12. Sorensen SV, Dewilde S, Singer DE, Goldhaber SZ, Monz BU, Plumb JM Cost-effectiveness of warfarin: trial versus "real-world" stroke prevention in atrial fibrillation. Am Heart J. 2009;157(6):1064-73.

13. Fang MC, Go AS, Chang Y, et al. Warfarin discontinuation after starting warfarin for atrial fibrillation. Circ Cardiovasc Qual Outcomes. 2010;3(6):624-31. Available at: http://circoutcomes.ahajournals.org/content/3/6/624.long. Accessed April 5, 2013.

14. van Walraven C, Hart RG, Singer DE, et al. Oral anticoagulants vs aspirin in nonvalvular atrial fibrillation: an individual patient meta-analysis. JAMA. 2002;288(19):2441-48. Available at: http://jama.jamanetwork.com/ article. aspx?articleid=195518. Accessed April 5, 2013.

15. Casciano JP, Singer DE, Kwong WJ, Fox ES, Martin BC. Anticoagulation therapy for patients with non-valvular atrial fibrillation: comparison of decision analytical model recommendations and real-world warfarin prescription use. Am J Cardiovasc Drugs. 2012;12(5):313-23.
16. Kim MH, Johnston SS, Chu BC, Dalal MR, Schulman KL. Estimation of total incremental health care costs in patients with atrial fibrillation in the United States. Circ Cardiovasc Qual Outcomes. 2011;4(3):313-20. Available at: http://circoutcomes.ahajournals.org/content/4/3/313.long. Accessed April 5, 2013.

17. Gage BF, Waterman AD, Shannon W, Boechler M, Rich MW, Radford MJ. Validation of clinical classification schemes for predicting stroke: results from the National Registry of Atrial Fibrillation. JAMA. 2001;285(22):286470. Available at: http://jama.jamanetwork.com/article. aspx?articleid=193912. Accessed April 5, 2013.

18. Fang MC, Go AS, Chang Y, et al. A new risk scheme to predict warfarinassociated hemorrhage: the ATRIA (Anticoagulation and Risk Factors in Atrial Fibrillation) Study. J Am Coll Cardiol. 2011;58(4)395-401. Available at: http:// www.ncbi.nlm.nih.gov/pmc/articles/PMC3175766/. Accessed April 5, 2013.

19. Gage BF, Yan Y, Milligan P, et al. Clinical classification schemes for predicting hemorrhage: results from the National Registry of Atrial Fibrillation (NRAF). Am Heart J. 2006;151(3):713-19.

20. Pisters R, Lane DA, Nieuwlaat R, de Vos CB, Crijns HJ, Lip GYH. A novel user-friendly score (HAS-BLED) to assess 1-year risk of major bleeding in patients with atrial fibrillation: the Euro Heart Survey. Chest. 2010;138(5):1093-100. Available at: http://journal.publications.chestnet.org/ article.aspx?articleid=1045174. Accessed April 5, 2013.

21. Go AS, Hylek EM, Chang Y, et al. Anticoagulation therapy for stroke prevention in atrial fibrillation: how well do randomized trials translate into clinical practice? JAMA. 2003;290(20):2685-92. Available at: http://jama. jamanetwork.com/article.aspx?articleid=197710. Accessed April 5, 2013.

22. Karve S, Cleves MA, Helm M, Hudson TJ, West DS, Martin BC. Good and poor adherence: optimal cut-point for adherence measures using administrative claims data. Curr Med Res Opin. 2009;25(9):2303-10.

23. Dodd S, Bassi A, Bodger K, Williamson P. A comparison of multivariable regression models to analyse cost data. J Eval Clin Pract. 2006;12(1):76-86.

24. Quan H, Sundararajan V, Halfon P, et al. Coding algorithms for defining comorbidities in ICD-9CM and ICD-10 administrative data. Med Care. 2005;43(11):1130-39.

25. Baker WL, Cios DA, Sander SD, Coleman CI. Meta-analysis to assess the quality of warfarin control in atrial fibrillation patients in the United States. J Manag Care Pharm. 2009;15(3):244-52. Available at: http://www.amcp.org/ data/jmcp/244-252.pdf.

26. Reynolds MR, Shah J, Essebag V, et al. Patterns and predictors of warfarin use in patients with new-onset atrial fibrillation from the FRACTAL Registry. Am J Cardiol. 2006;97(4):538-43. 
The Costs of Warfarin Underuse and Nonadherence in Patients with Atrial Fibrillation: A Commercial Insurer Perspective

\section{APPENDIX A ICD-9-CM Diagnoses for Excluding Patients with Valvular Disease}

\begin{tabular}{|c|c|}
\hline Measure & ICD-9-CM Code \\
\hline Closed heart valvotomy, unspecified valve & 35 \\
\hline Closed heart valvotomy, aortic valve & 35.01 \\
\hline Closed heart valvotomy, mitral valve & 35.02 \\
\hline Closed heart valvotomy, pulmonary valve & 35.03 \\
\hline Closed heart valvotomy, tricuspid valve & 35.04 \\
\hline $\begin{array}{l}\text { Open heart valvuloplasty of unspecified valve, without } \\
\text { replacement }\end{array}$ & 35.1 \\
\hline $\begin{array}{l}\text { Open heart valvuloplasty of aortic valve, without } \\
\text { replacement }\end{array}$ & 35.11 \\
\hline $\begin{array}{l}\text { Open heart valvuloplasty of mitral valve, without } \\
\text { replacement }\end{array}$ & 35.12 \\
\hline $\begin{array}{l}\text { Open heart valvuloplasty of pulmonary valve, without } \\
\text { replacement }\end{array}$ & 35.13 \\
\hline $\begin{array}{l}\text { Open heart valvuloplasty of tricuspid valve, without } \\
\text { replacement }\end{array}$ & 35.14 \\
\hline Replacement of unspecified heart valve & 35.2 \\
\hline Replacement of aortic valve with tissue graft & 35.21 \\
\hline Other replacement of aortic valve & 35.22 \\
\hline Replacement of mitral valve with tissue graft & 35.23 \\
\hline Other replacement of mitral valve & 35.24 \\
\hline Replacement of pulmonary valve with tissue graft & 35.25 \\
\hline Other replacement of pulmonary valve & 35.26 \\
\hline Replacement of tricuspid valve with tissue graft & 35.27 \\
\hline Other replacement of tricuspid valve & 35.28 \\
\hline Mitral stenosis & 394 \\
\hline Mitral stenosis with insufficiency & 394.2 \\
\hline Mitral valve stenosis and aortic valve stenosis & 396 \\
\hline Mitral valve stenosis and aortic valve insufficiency & 396.1 \\
\hline Multiple involvement of mitral and aortic valves & 396.8 \\
\hline Organ or tissue replaced by transplant & $\mathrm{V} 42$ \\
\hline Heart valve & $\mathrm{V} 42.2$ \\
\hline Organ or tissue replaced by other means & $\mathrm{V} 43 \mathrm{O}$ \\
\hline Heart valve & V43.3 \\
\hline Operations of structures adjacent to heart valves & 35.3 \\
\hline Operations on papillary muscle & 35.31 \\
\hline Operations on chordae tendinaea & 35.32 \\
\hline Annuloplasty & 35.33 \\
\hline Infundibulectomy & 35.34 \\
\hline Operations on trabeculae carness cordis & 35.35 \\
\hline $\begin{array}{l}\text { Operations on other structures adjacent to valves of } \\
\text { heart }\end{array}$ & 35.39 \\
\hline Production of septal defect in heart & 35.4 \\
\hline
\end{tabular}

\begin{tabular}{|c|c|}
\hline Measure & ICD-9-CM Code \\
\hline Enlargement of existing atrial septal defect & 35.41 \\
\hline Creation of septal defect in heart & 35.42 \\
\hline Repair of atrial and ventricular septa with prosthesis & 35.5 \\
\hline $\begin{array}{l}\text { Repair of unspecified septal defect of heart with } \\
\text { prosthesis }\end{array}$ & 35.5 \\
\hline $\begin{array}{l}\text { Repair of atrial septal defect with prosthesis open } \\
\text { technique }\end{array}$ & 35.51 \\
\hline $\begin{array}{l}\text { Repair of atrial septal defect with prosthesis, closed } \\
\text { technique }\end{array}$ & 35.52 \\
\hline Repair of ventricular septal defect with prosthesis & 35.53 \\
\hline Repair of endocardial cushion defect with prosthesis & 35.54 \\
\hline Repair of atrial and ventricular septa with tissue graft & 35.6 \\
\hline $\begin{array}{l}\text { Repair of unspecified septol defect of heart } \\
\text { with tissue graft }\end{array}$ & 35.6 \\
\hline Repair of atrial septal detect with tissue graft & 35.61 \\
\hline Repair of ventricular septal defect with tissue graft & 35.62 \\
\hline Repair of endocardial cushion defect with tissue graft & 35.63 \\
\hline $\begin{array}{l}\text { Other and unspecified repair of unspecified specific } \\
\text { defect of heart }\end{array}$ & 35.7 \\
\hline Other and unspecified repair of atrial septal defect & 35.71 \\
\hline $\begin{array}{l}\text { Other and unspecified repair of ventricular } \\
\text { septal defect }\end{array}$ & 35.72 \\
\hline $\begin{array}{l}\text { Other and unspecified repair of endocardial cushion } \\
\text { defect }\end{array}$ & 35.73 \\
\hline Bypass anastomosis for heart revascularization & 36.1 \\
\hline Aortocoronary bypass of one coronary artery & 36.11 \\
\hline Aortocoronary bypass of two coronary arteries & 36.12 \\
\hline Aortocoronary bypass of three coronary arteries & 36.13 \\
\hline Aortocoronary bypass of four or more coronary arteries & 36.14 \\
\hline Single internal mammory-coronary artery bypass & 36.15 \\
\hline Double internal mammary-coronary artery bypass & 36.16 \\
\hline Abdominal-coronary artery bypass & 36.17 \\
\hline Other bypass anastomosis for heart revascularization & 36.19 \\
\hline Cardiotomy and pericardiotomy & 37.1 \\
\hline incision of heart, not otherwise specified & 37.1 \\
\hline Cardiotomy & 37.11 \\
\hline Pericardiotomy & 37.12 \\
\hline Periocardiectomy and excision of lesion of heart & 37.3 \\
\hline Pericardiotomy & 37.31 \\
\hline Excision of aneurysm of heart & 37.32 \\
\hline Excision el destruction of other lesion or tissue of heart & 37.33 \\
\hline Repair of heart and pericardium & 37.4 \\
\hline
\end{tabular}

ICD-9-CM = International Classification of Diseases, Ninth Revision, Clinical Modification. 
The Costs of Warfarin Underuse and Nonadherence in Patients with Atrial Fibrillation: A Commercial Insurer Perspective

\section{APPENDIX B ICD-9-CM Codes Used to Identify Contraindication to Warfarin}

\begin{tabular}{|c|c|c|c|}
\hline Measure & ICD-9-CM Code & Measure & ICD-9-CM Code \\
\hline Pregnancy & $\begin{array}{l}\text { V22.0-.2 } \\
650\end{array}$ & \multirow[t]{9}{*}{$\begin{array}{l}\text { Acquired immunodeficiency syndrome (human immu- } \\
\text { nodeficiency virus infection) }\end{array}$} & $\begin{array}{r}042 \\
0420\end{array}$ \\
\hline Blood dyscrasias & 289.9 & & 0421 \\
\hline $\begin{array}{l}\text { Recent or complicated surgery of: } \\
\text { CNS } \\
\text { Eye }\end{array}$ & $\begin{array}{l}\text { V58.72 } \\
\text { V45.6 }\end{array}$ & & $\begin{array}{l}0422 \\
0429 \\
0430\end{array}$ \\
\hline Trauma surgery resulting in large open surface area & V58.43 & & $\begin{array}{l}0431 \\
0432\end{array}$ \\
\hline $\begin{array}{l}\text { Bleeding tendencies associated with } \\
\text { active ulceration or overt bleeding of: } \\
\text { Gastrointestinal/Genito-Urinary/Respiratory tract }\end{array}$ & $\begin{array}{l}578.9 \\
627.1\end{array}$ & & $\begin{array}{l}0433 \\
0439 \\
0440\end{array}$ \\
\hline Cerebrovascular hemorrhage & $\begin{array}{c}430 \\
431 \\
432.0, .1, .9 \\
\end{array}$ & & $\begin{array}{l}0449 \\
079.53 \\
279.10\end{array}$ \\
\hline Aneurysm-cerebral & 437.3 & & 279.19 \\
\hline Dissecting aorta & $441.00-.03$ & & 795.71 \\
\hline \multirow[t]{2}{*}{ Pericarditis and pericardial effusion } & $\begin{array}{l}420.0 \\
4209\end{array}$ & & V08 \\
\hline & $\begin{array}{r}420.9 \\
423.9 \\
4210\end{array}$ & Dementia & $\begin{array}{l}294.10 \\
294.11\end{array}$ \\
\hline Bacterial endocarditis & 421.0 & \multirow[t]{10}{*}{ Liver disease } & 571.5 \\
\hline Threatened abortion & 640.0 & & 572.0 \\
\hline Eclampsia/pre-eclampsia & $\begin{array}{l}642.4 \\
642.6 \\
642.7\end{array}$ & & $\begin{array}{l}572.1 \\
572.2 \\
572.3\end{array}$ \\
\hline Hypersensitivity to warfarin & 995.27 & & 572.8 \\
\hline History of cirrhosis & $\begin{array}{l}571.5 \\
571.2\end{array}$ & & $\begin{array}{l}789.5 \\
789.59\end{array}$ \\
\hline Hepatitis & $\begin{array}{l}571.40 \\
571.41\end{array}$ & & $\begin{array}{l}570 \\
571.6\end{array}$ \\
\hline Seizure disorder & $\begin{array}{l}345.8 \\
345.9 \\
780.39\end{array}$ & & $\begin{array}{l}571.8 \\
571.9 \\
573.0 \\
573.4\end{array}$ \\
\hline Previous intracranial hemmorhage & 432.9 & & 573.8 \\
\hline Diagnostic spinal tap/other diagnostic procedure & $\begin{array}{l}03.31 \\
03.32 \\
03.39 \\
\end{array}$ & & $\begin{array}{l}573.9 \\
782.4 \\
789.1 \\
7904\end{array}$ \\
\hline Alcohol-related disorders & $\begin{array}{c}\text { Same } \\
\text { codes as } \\
\text { Appendix E }\end{array}$ & & $\begin{array}{l}790.4 \\
790.5 \\
\text { V42.8 }\end{array}$ \\
\hline
\end{tabular}

\section{APPENDIX C ICD-9-CM Diagnoses for $\mathrm{CHADS}_{2}$} Stroke Risk Factors

\begin{tabular}{l|l}
\hline Prior stroke & $433 . x x-434 . x x$ \\
\hline Transient cerebral ischemia & $435.0,435.1,435.2,435.3,435.8,435.9$ \\
\hline Hypertension & $401 . x x-405 . x x$ \\
\hline Diabetes & $250.0 x-250.8 x$ \\
\hline Expanded diabetes & $249 . x x, 250.9 x, 790.2,790.21,790.22$, \\
& $790.29,791.5,791.6$ \\
\hline Heart failure & $398.91,402.01,402.11,402.91$, \\
& $428.0 x-428.9 x, 518.4$
\end{tabular}

ICD-9-CM = International Classification of Diseases, Ninth Revision, Clinical

Modification. 
The Costs of Warfarin Underuse and Nonadherence in Patients with Atrial Fibrillation: A Commercial Insurer Perspective

\section{APPENDIX D ICD-9-CM Diagnoses for ATRIA Bleeding Risk Factors}

Description

ICD-9-CM Code

Anemia

Acute posthemorrhagic anemia

Sickle cell anemia

285.1

$282.41,282.42,282.5,282.60,282.61,282.62,282.63$

$282.64,282.68,282.69$

Deficiency and other anemia

Iron deficiency anemia

Other deficiency anemia

Aplastic anemia

Chronic blood loss anemia

Acquired hemolytic anemia

Other specified anemia

Anemia; unspecified

Renal failure

Acute and unspecified renal failure

Acute renal failure

Unspecified renal failure

Chronic renal failure

$280.1,280.8,280.9$

$281.0,281.1,281.2,281.3,281.4,281.8,281.9$

$284.0,284.01,284.09,284.1,284.8,284.81,284.89$,

284.9

280.0

283.0, 283.1, 283.10, 283.11, 283.19, 283.2, 283.9

282.0, 282.1, 282.2, 282.3, 282.4, 282.49, 282.7, 282.8,

$282.9,284.2,285.0,285.21,285.22,285.29,285.8$

285.9

$584.5,584.6,584.7,584.8,584.9$

586

585, 585.3, 585.4, 585.5, 585.6, 585.9, 792.5, V42.0,

V45.1, V45.11, V45.12, V56.0, V56.1, V56.2, V56.31,

V56.32, V56.8

History of any bleeding

Prior intracranial hHemorrhage

Prior gastrointestinal hemorrhage

Internal hemorrhoids with other complication

430.xx, 431.xx, 432.x, 852.0x, 852.2x, 852.4x, 853.0x

External hemorrhoids with other complication

Unspecified hemorrhoids with other complication

Esophageal varicies with bleeding

Esophageal varicies in diseases classified elsewhere with bleeding

Hemorrhage, unspecified

Gastroesophageal laceration-hemorrhage syndrome

Esophageal hemorrhage

Acute gastric ulcer with hemorrhage without mention of obstruction

Acute gastric ulcer with hemorrhage with obstruction

Acute gastric ulcer with hemorrhage and perforation without mention of obstruction

Acute gastric ulcer with hemorrhage and perforation with obstruction

455.2

Chronic or unspecified gastric ulcer with hemorrhage without mention of obstruction

Chronic or unspecified gastric ulcer with hemorrhage with obstruction

Chronic or unspecified gastric ulcer with hemorrhage and perforation without mention of

obstruction

Chronic or unspecified duodenal ulcer with hemorrhage and perforation with obstruction

Acute peptic ulcer, site unspecified, with hemorrhage without mention of obstruction

Acute peptic ulcer, site unspecified, with hemorrhage with obstruction

Acute peptic ulcer, site unspecified, with hemorrhage and perforation without mention of obstruction

Acute peptic ulcer, site unspecified, with hemorrhage and perforation with obstruction

Chronic or unspecified peptic ulcer, site unspecified, with hemorrhage without mention of obstruction

Chronic or unspecified peptic ulcer, site unspecified, with hemorrhage with obstruction

Chronic or unspecified peptic ulcer, site unspecified, with hemorrhage and perforation

Acute gastrojejunal ulcer with hemorrhage without mention of obstruction

Acute gastrojejunal ulcer with hemorrhage with obstruction

Acute gastrojejunal ulcer with hemorrhage and perforation without mention of obstruction

Acute gastrojejunal ulcer with hemorrhage and perforation with obstruction

Chronic or unspecified gastrojejunal ulcer with hemorrhage without mention of obstruction

Chronic or unspecified gastrojejunal ulcer with hemorrhage with obstruction

455.2

455.5

455.8

456.0

456.20

459.0

530.7

530.82

531.00

531.01

531.20

531.21

531.40

531.41

531.60

531.61

533.00

533.01

533.20

533.21

533.40

533.41

533.61

534.00

534.01

534.20

534.21

534.40

534.41 


\section{APPENDIX D ICD-9-CM Diagnoses for ATRIA Bleeding Risk Factors (continued)}

Description

Chronic or unspecified gastrojejunal ulcer with hemorrhage and perforation with obstruction Acute gastritis with hemorrhage

Atrophic gastritis with hemorrhage

Gastric mucosal hypertrophy with hemorrhage

Alcoholic gastritis with hemorrhage

Other specified gastritis with hemorrhage

Unspecified gastritis and gastroduodenitis with hemorrhage

Duodenitis with hemorrhage

Angiodysplasia of stomach and duodenum with hemorrhage (added December 4, 2000)

Diverticulosis of small intestine with hemorrhage

Diverticulitis of small intestine with hemorrhage

Diverticulosis of colon with hemorrhage

Diverticulitis of colon with hemorrhage

Hemoperitoneum (nontraumatic)

Hemorrhage of rectum and anus

Angiodysplasia of intestine with hemorrhage

Gastrointestinal hemorrhage

Hematemesis

Blood in stool

Hemorrhage of gastrointestinal tract, unspecified

Other prior hemorrhage

Hemopericardium

Hemorrhage, unspecified

Vascular disorders of the kidney

Hemarthrosis

Hemarthrosis: site unspecified

Hemarthrosis: shoulder region

Hemarthrosis: upper arm

Hemarthrosis: forearm

Hemarthrosis: hand

Hemarthrosis: pelvic region and thigh

Hemarthrosis: lower leg

Hemarthrosis: ankle and foot

Hemarthrosis: other specified sites

Hemarthrosis: multiple sites

Epistaxis

Hemorrhage from throat (added December 4, 2000)

Hemoptysis

Hematuria

ICD-9-CM = International Classification of Diseases, Ninth Revision, Clinical Modification.
ICD-9-CM Code

534.61

535.01

535.11

535.21

535.31

535.41

535.51

535.61

537.83

562.02

562.03

562.12

562.13

568.81

569.3

569.85

578

578.0

578.1

578.9

423.0

459.0

593.81

719.1

719.10

719.11

719.12

719.13

719.14

719.15

719.16

719.17

719.18

719.19

784.7

784.8

786.3

599.7 
The Costs of Warfarin Underuse and Nonadherence in Patients with Atrial Fibrillation: A Commercial Insurer Perspective

\section{APPENDIX E ICD-9-CM Diagnoses for HEMORR ${ }_{2}$ HAGES Bleeding Risk Factors}

\begin{tabular}{|c|c|}
\hline Description & ICD-9-CM Code \\
\hline Anemia & Same codes as used in the ATRIA risk scheme (Appendix D) \\
\hline Ethanol abuse & $\begin{array}{l}\text { 291.0, 291.1, 291.2, 291.3, 291.4, 291.5, 291.8, 291.81, 291.82, 291.89, 291.9, 303.00, 0301, 303.02, 303.03, } \\
303.90,303.91,303.92,0393,305.00,305.01,305.02,305.03,760.71,980.0\end{array}$ \\
\hline Prior bleed & Same codes as used in the ATRIA risk scheme (Appendix D) \\
\hline Hypertension & Same codes as used in the $\mathrm{CHADS}_{2}$ risk scheme (Appendix C) \\
\hline Stroke & Same codes as used in the CHADS $_{2}$ risk scheme (Appendix C) \\
\hline \multicolumn{2}{|l|}{ Hepatic or renal disease } \\
\hline Acute renal failure & $584.5,584.6,584.7,584.8,584.9$ \\
\hline Unspecified renal failure & 586 \\
\hline Chronic renal failure & $\begin{array}{l}\text { 585, 585.3, 585.4, 585.5, 585.6, 585.9, 792.5, V42.0, V45.1, V45.11, V45.12, V56.0, V56.1, V56.2, V56.31 } \\
\text { V56.32, V56.8 }\end{array}$ \\
\hline Cirrhosis of liver without mention of alcohol & 571.5 \\
\hline $\begin{array}{l}\text { Liver abscess and sequelae of chronic liver } \\
\text { disease }\end{array}$ & $572.0,572.1,572.2,572.3,572.4,572.8$ \\
\hline Ascites & $789.5,789.59$ \\
\hline Other and unspecified liver disorders & $570,571.6,571.8,571.9,573.0,573.4,573.8,573.9,782.4,789.1,790.4,790.5,794.8$, V42.7 \\
\hline \multicolumn{2}{|l|}{ Reduced platelet function } \\
\hline Thrombocytopenia & $287.3,287.30,287.31,287.32,287.33,287.39,287.4,287.49,287.5,289.84$ \\
\hline Malignancy & $153.0-229.9$ \\
\hline Genetic factors & Not available in the dataset so will be scored as 0. \\
\hline \multicolumn{2}{|l|}{ Excessive fall risk } \\
\hline $\begin{array}{l}\text { Delirium dementia and amnestic and other } \\
\text { cognitive disorders }\end{array}$ & $\begin{array}{l}290.0,290.10,290.11,290.12,290.13,290.20,290.21,290.3,290.40,290.41,290.42,290.43,290.8,290.9, \\
\text { 293.0, 293.1, 294.0, 294.1, 294.10, 294.11, 294.8, 294.9, 310.0, 310.2, 310.8, 310.9, 331.0, 331.1, 331.11, } \\
\text { 331.19, 331.2, 331.8, } 797\end{array}$ \\
\hline Schizophrenia and other psychotic disorders & 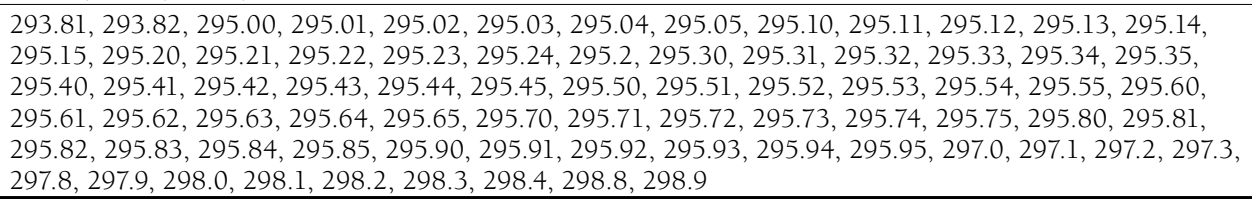 \\
\hline
\end{tabular}

CD-9-CM=International Classification of Diseases, Ninth Revision, Clinical Modification

\section{APPENDIX F ICD-9-CM Diagnoses for HAS-BLED} Bleeding Risk Factors

\begin{tabular}{l|l}
\hline Hypertension & $\begin{array}{l}\text { Same codes as used in the } \mathrm{CHADS}_{2} \text { risk scheme } \\
\text { (Appendix C) }\end{array}$ \\
\hline Ethanol abuse & $\begin{array}{l}\text { Same codes as used in HEMORR } \\
\text { (Appendix E) }\end{array}$ \\
\hline Stroke & $\begin{array}{l}\text { Same codes as used in the } \mathrm{CHADS}_{2} \text { risk scheme } \\
\text { (Appendix C) }\end{array}$ \\
\hline Prior bleed & $\begin{array}{l}\text { Same codes as used in the ATRIA risk scheme } \\
\text { (Appendix D), also including anemia codes }\end{array}$ \\
\hline LABILE INRs & $\begin{array}{l}\text { Not available in the data; will be assumed to be 0 for } \\
\text { all patients }\end{array}$ \\
\hline $\begin{array}{l}\text { Abnormal renal or } \\
\text { liver function }\end{array}$ & $\begin{array}{l}\text { Same codes as used in HEMORR } \\
\text { (Appendix E) }\end{array}$ \\
\hline $\begin{array}{l}\text { ICD-9-CM }=\text { International Classification of Diseases, Ninth Revision, Clinical } \\
\text { Modification; INR=international normalized rate. }\end{array}$
\end{tabular}

